March 1999

JINR E2-99-43

SHEP/99/01

\title{
Gribov copies and gauge variant correlators in $U(1)$ lattice gauge theory
}

\author{
I. L. Bogolubsky ${ }^{1}$, L. Del Debbio ${ }^{2}$ and V.K. Mitrjushkin ${ }^{1}$ \\ ${ }^{1}$ Joint Institute for Nuclear Research, Dubna, Russia \\ ${ }^{2}$ Dept. of Physics and Astronomy, Univ. of Southampton SO17 1BJ, UK
}

\begin{abstract}
We discuss the influence of Dirac sheets and zero-momentum modes on the gauge variant photon correlators $\Gamma(\tau ; \vec{p})$ with $\vec{p} \neq 0$ and $\vec{p}=0$ in the pure gauge $U(1)$ theory. A special attention has been paid to the $\beta$ - and volume-dependence of this influence. Numerical simulations are performed on $12 \times 6^{3}$ and $24 \times 12^{3}$ lattices at different $\beta$ 's in the Coulomb phase.
\end{abstract}

\section{Introduction}

Lattice approach [1] gives a possibility to calculate gauge invariant objects without gauge fixing. However, numerical calculations of gauge dependent objects, e.g., gauge field correlators $\Gamma(\tau ; \vec{p})$, can also be of interest. For example, the comparison of gauge variant objects with that calculated perturbatively can provide some deeper insight into the structure of the lattice theory. In particular, the role of lattice artifacts and/or Gribov copies [2] can be investigated.

Compact $U(1)$ pure gauge theory in the Coulomb phase provides a unique 'test ground' for the lattice approach. Indeed, in the weak coupling limit one expects to find a trivial theory of the free non-interacting photons. Therefore, at sufficiently small coupling a gauge variant transverse correlator $\Gamma_{T}(\tau ; \vec{p})$ is supposed to fit the perturbative expression given in eq.(3.5).

A few years ago it has been shown [3] that in the Coulomb phase some of the gauge copies produce a photon correlator $\Gamma_{T}(\tau ; \vec{p} \neq 0)$ with a decay behavior 
inconsistent with perturbation theory. Numerical study [4] has shown that there is a connection between 'bad' gauge copies and the appearance of configurations with periodically closed double Dirac sheets (DDS). The explanation of this effect [5] is connected with a nontrivial 'vacuum' structure of the classical compact $U(1)$ theory, i.e. with the existence of the nontrivial stable classical solutions ('vacua'), some of these solutions - DDS - being Gribov copies of the trivial 'vacuum' with zero field.

Another interesting observation is connected with the $\tau$-dependence of the zeromomentum gauge variant correlators $\Gamma(\tau)$. The analysis of the $\tau$-dependence of the correlator $\Gamma(\tau)$ in the $S U(3)$ lattice gauge theory drove the authors of [6] to the conclusion that $\Gamma(\tau)$ is consistent with the propagation of a massive gluon. On the other side, in the $U(1)$ theory the correlator $\Gamma(\tau)$ exhibits a similar behaviour

[7]. However, one can hardly expect the appearance of the massive photon in the Coulomb phase in the $U(1)$ theory. It has been shown in ref. [8] that (at least, in the case of the $U(1)$ theory) the $\tau$-dependence of the correlator $\Gamma(\tau)$ can be explained taking into account zero-momentum mode of the gauge field. The influence of the zero-momentum modes on the gluon propagators in nonabelian theories has been pointed in [9, 10, 11.

In this paper we discuss the effects connected with Dirac sheets and zeromomentum modes in more details. The main questions addressed in this paper are the following.

- What is the $\beta$-dependence of the frequency of the Dirac sheets appearance ? Does the contribution of the Dirac sheets to the gauge variant correlator $\Gamma(\tau ; \vec{p})$ become more (or less) important when the coupling $\beta$ is increased?

- What is the volume dependence of the correlator $\Gamma_{T}(\tau ; \vec{p})$ ? Does the influence of DDS become smaller in the thermodynamical limit?

- What is the influence of zero-momentum modes on the zero-momentum correlator $\Gamma(\tau)$ ? How does this influence depend on $\beta$ and lattice size ?

Throughout this paper a $4 d$ lattice with periodic boundary conditions is considered. $N_{\mu}$ is the lattice size in the direction $\mu$, and $V_{4}=N_{1} N_{2} N_{3} N_{4}$. The lattice derivatives are $\partial_{\mu} f(x)=f(x+\hat{\mu})-f(x)$ and $\bar{\partial}_{\mu} f(x)=f(x)-f(x-\hat{\mu})$, and the lattice spacing is chosen to be unity.

\section{The action and classical solutions}

The standard Wilson action $S(U)$ is

$$
S(U)=\beta \sum_{x} \sum_{\mu>\nu}\left(1-\cos \theta_{x ; \mu \nu}\right)
$$


where $U_{x \mu}=e^{i \theta_{x \mu}} \in U(1)$ are link variables, $\theta_{x ; \mu \nu}=\partial_{\mu} \theta_{x \nu}-\partial_{\nu} \theta_{x \mu}$ are the plaquette angles and $\beta=1 / g^{2}$. This action is the part of the full QED action $S_{Q E D}$, which is supposed to be compact if we consider QED as arising from a subgroup of a non-abelian (e.g., grand unified) gauge theory [12].

The plaquette angle $\theta_{P} \equiv \theta_{x ; \mu \nu}$ can be split up: $\theta_{P}=\left[\theta_{P}\right]+2 \pi n_{P}$, where $\left[\theta_{P}\right] \in(-\pi ; \pi]$ and $n_{P}=0, \pm 1, \pm 2$. The plaquettes with $n_{P} \neq 0$ are called Dirac plaquettes. The dual integer valued plaquettes $m_{x, \mu \nu}=\frac{1}{2} \varepsilon_{\mu \nu \rho \sigma} n_{x, \rho \sigma}$ form Dirac sheets 13.

In a perturbative language the existence of the gauge copies can be interpreted as a problem of the gauge copies of the 'vacuum' configurations, i.e. the solutions of the classical equations of motion. Assuming that every configuration is some small fluctuation about the corresponding 'vacuum' one can find all gauge copies of this configuration provided all gauge copies of the 'vacuum' are known.

The classical equations of motion are

$$
\sum_{\nu} \bar{\partial}_{\nu} \sin \theta_{x ; \mu \nu}^{c l}=0
$$

Evidently, zero-momentum modes $\theta_{x \mu}^{c l}=\phi_{\mu}$ are the solutions of these equations.

Choosing the Lorentz (or Landau) gauge $\sum_{\mu} \bar{\partial}_{\mu} \theta_{x \mu}=0$, one can write expicitely a solution corresponding to a single Dirac sheet [5]

$$
\begin{aligned}
& \theta_{x 1}^{c l}(\vec{R})=\frac{2 \pi i}{N_{1} N_{2}} \sum_{\vec{q}_{\perp} \neq 0} \frac{\mathcal{K}_{2}}{\overrightarrow{\mathcal{K}}_{\perp}^{2}} \cdot e^{i \vec{q}_{\perp}\left(\vec{x}_{\perp}-\vec{R}\right)-\frac{i}{2} q_{2}} ; \\
& \theta_{x 2}^{c l}(\vec{R})=-\frac{2 \pi i}{N_{1} N_{2}} \sum_{\vec{q}_{\perp} \neq 0} \frac{\mathcal{K}_{1}}{\overrightarrow{\mathcal{K}}_{\perp}^{2}} \cdot e^{i \vec{q}_{\perp}\left(\vec{x}_{\perp}-\vec{R}\right)-\frac{i}{2} q_{1}},
\end{aligned}
$$

where $\mathcal{K}_{\mu}=2 \sin \frac{q_{\mu}}{2}$ and $\overrightarrow{\mathcal{K}}_{\perp}^{2}=\mathcal{K}_{1}^{2}+\mathcal{K}_{2}^{2}$. The two-dimensional vector $\vec{R}=\left(R_{1} ; R_{2}\right)$ corresponds to the position of the Dirac plaquette in the $\left(x_{1} ; x_{2}\right)$ plane:

$$
\theta_{x ; 12}^{c l}=2 \pi \cdot \delta_{\vec{x}_{\perp} ; \vec{R}}-\Delta ; \quad \Delta=\frac{2 \pi}{N_{1} N_{2}} .
$$

Of course, $\theta_{x \mu}^{\prime}=\phi_{\mu}+\theta_{x \mu}^{c l}$ is also a solution of eq's.(2.2).

The single Dirac sheet solution $\theta_{x \mu}^{c l}(\vec{R})$ corresponds to a local minimum of the action, i.e. that it is stable with respect to small fluctuations. The existence of the long-living metastable states corresponding to single Dirac sheets was observed in simulations in the pure gauge $U(1)$ theory [14].

The classical gauge action is $S\left(\theta^{c l}\right)=\frac{2 V_{4}}{g^{2}}(1-\cos \Delta)$. On a symmetric lattice $N_{1}=N_{2}=N_{3}=N_{4} \rightarrow \infty$ the action $S\left(\theta^{c l}\right)$ is non-zero and finite : 


$$
S\left(\theta^{c l}\right)=\frac{4 \pi^{2}}{g^{2}}<\infty .
$$

Double Dirac sheet solution of the classical equation of motion consists of the two single Dirac sheets with an opposite orientation of the flux :

$$
\theta_{x i}^{c l}\left(\vec{R}^{a} ; \vec{R}^{b}\right)=\theta_{x i}^{c l}\left(\vec{R}^{a}\right)-\theta_{x i}^{c l}\left(\vec{R}^{b}\right), \quad i=1 ; 2,
$$

where the vectors $\vec{R}^{a}$ and $\vec{R}^{b}$ correspond to the two Dirac plaquettes in the plane $\left(x_{1} ; x_{2}\right)$. It is easy to see that

$$
\theta_{x ; 12}^{c l}\left(\vec{R}^{a} ; \vec{R}^{b}\right)=2 \pi \cdot\left[\delta_{\vec{x}_{\perp} ; \vec{R}^{a}}-\delta_{\vec{x}_{\perp} ; \vec{R}^{b}}\right] .
$$

The double Dirac sheet $\theta_{x ; i}^{c l}\left(\vec{R}^{a} ; \vec{R}^{b}\right)$ has a zero action: $S\left(\theta^{c l}\right)=0$.

Gauge transformations can shift the Dirac sheets and change their form. For example, the 'big' gauge transformation function $\Omega_{x}$

$$
\Omega_{x}=-\frac{2 \pi}{N_{1} N_{2}} \sum_{\vec{q}_{\perp} \neq 0} \frac{e^{i \vec{q}_{\perp}\left(\vec{x}_{\perp}-\vec{R}\right)}}{\overrightarrow{\mathcal{K}}_{\perp}^{2}} \cdot\left(1-e^{-i q_{2}}\right) ;
$$

shifts the single Dirac sheet in the $x_{1}$-direction. The gauge transformation $\Omega_{x}$ in eq.(2.7) applied to the zero-field creates a double Dirac sheet as in eq.(2.5) with $\vec{R}^{a}=\vec{R}$ and $\vec{R}^{b}=\vec{R}-\hat{1}$. Therefore, $\theta_{x ; i}^{c l}\left(\vec{R}^{a} ; \vec{R}^{b}\right)$ is a Gribov copy of the zero solution $\theta_{x \mu}^{c l}=0$. It is not difficult now to obtain general Dirac sheet solutions, i.e. the Dirac sheets curved in the four-dimensional space [0]].

\section{$3 \quad \Gamma_{T}(\tau ; \vec{p})$ and Dirac sheets}

In lattice calculations the usual choice of the Lorentz (or Landau) gauge is

$$
\sum_{\mu=1}^{4} \bar{\partial}_{\mu} \sin \theta_{x \mu}=0
$$

which is equivalent to finding extremum of the functional $F(\theta)$

$$
F(\theta)=\frac{1}{V_{4}} \sum_{x} F_{x}(\theta) ; \quad F_{x}(\theta)=\frac{1}{8} \sum_{\mu=1}^{4}\left[\cos \theta_{x \mu}+\cos \theta_{x-\hat{\mu} ; \mu}\right]
$$

with respect to gauge transformations $U_{x \mu} \longrightarrow \Lambda_{x} U_{x \mu} \Lambda_{x+\mu}^{*} ; \Lambda_{x}=\exp \left\{i \Omega_{x}\right\}$. It is important to note that all link angles $\theta_{x \mu}$ are compact variables $\left(-\pi<\theta_{x \mu} \leq \pi\right)$, and the gauge transformations $\theta_{x \mu} \stackrel{\Omega}{\rightarrow} \theta_{x \mu}-\partial_{\mu} \Omega_{x}$ are understood modulo $2 \pi$.

To find extremum of $F(\theta)$ we carried out a standard overrelaxation gauge cooling procedure with parameter $\alpha$ tuned to minimize the number of the cooling 
steps for given $\beta$ and volume. In our computations values of $\alpha$ were chosen to be between $\alpha=1.66$ and $\alpha=1.84$. The stopping criterion was

$$
\max \left|\sum_{\mu} \bar{\partial}_{\mu} \sin \theta_{x \mu}\right|<10^{-5} \text { and } \frac{1}{V} \sum_{x}\left|\sum_{\mu} \bar{\partial}_{\mu} \sin \theta_{x \mu}\right|<10^{-6} .
$$

The photon correlator $\Gamma_{\mu}(\tau ; \vec{p})$ is

$$
\Gamma_{\mu}(\tau ; \vec{p})=\left\langle\mathcal{O}_{\mu}^{*}(\tau ; \vec{p}) \mathcal{O}_{\mu}(0 ; \vec{p})\right\rangle=\frac{1}{N_{4}} \sum_{t=0}^{N_{4}-1}\left\langle\mathcal{O}_{\mu}^{*}(t \oplus \tau ; \vec{p}) \mathcal{O}_{\mu}(t ; \vec{p})\right\rangle
$$

where $t \oplus \tau=(t+\tau) \bmod N_{4}$ and

$$
\mathcal{O}_{\mu}(\tau ; \vec{p})=\sum_{\vec{x}} e^{-i \vec{p} \vec{x}-\frac{i}{2} p_{\mu}} \cdot \sin \theta_{x \mu}, \quad \mu=1,2,3
$$

and, clearly, $\left\langle O_{\mu}\right\rangle=0$.

Let us choose the momentum $\vec{p}=\left(0 ; p_{2} ; 0\right)$ with $p_{2} \neq 0$, and $\mu=1$. The perturbative expansion about the zero solution of the classical equation of motion $\theta_{x \mu}^{c l}=0$, i.e. the standard perturbation theory, gives in the lowest approximation

$$
\Gamma_{1}^{p e r t}(\tau ; \vec{p}) \sim e^{-\tau E_{p}}+e^{-\left(N_{4}-\tau\right) E_{p}}
$$

where the energy $E_{p}$ satisfies the lattice dispersion relation

$$
\sinh ^{2} \frac{E_{p}}{2}=\sum_{i=1}^{3} \sin ^{2} \frac{p_{i}}{2}
$$

It is easy to see that the expansion about a Dirac sheet solution gives a contribution to the correlator very different from that in eq.(3.5).

As an example, let us choose the flat double Dirac sheet with the space-like Dirac plaquettes $\theta_{x \mu}^{c l}\left(\vec{R}_{1} ; \vec{R}_{2}\right)$ as defined in eq.(2.5). In this case the correlator in the double Dirac sheet background is

$$
\Gamma_{1}^{d d s}(\tau ; \vec{p})=|\Phi(\vec{p})|^{2}+\frac{g^{2}}{2 V_{3}} \sum_{\vec{q}_{\perp}} G\left(\tau ; \vec{q}_{\perp}+\vec{p}\right) \cdot\left|\Psi\left(\vec{q}_{\perp}\right)\right|^{2}
$$

where

$$
\Phi(\vec{q})=\frac{\delta_{q_{3} ; 0}}{N_{1} N_{2}} \sum_{\vec{x}_{\perp}} e^{-i \vec{q}_{\perp} \vec{x}_{\perp}} \sin \theta_{x 1}^{c l} ; \quad \Psi(\vec{q})=\frac{\delta_{q_{3} ; 0}}{N_{1} N_{2}} \sum_{\vec{x}_{\perp}} e^{-i \vec{q}_{\perp} \vec{x}_{\perp}} \cos \theta_{x 1}^{c l}
$$

and 


$$
\begin{aligned}
G(\tau ; \vec{q}) & =\left[1+\frac{\mathcal{K}_{1}^{2}(q)}{2 \sinh E_{q}} \cdot \frac{d}{d E_{q}}\right] G_{0}(\tau ; \vec{q}) ; \\
G_{0}(\tau ; \vec{q}) & =\frac{1}{2 \sinh E_{q}} \frac{1}{1-e^{-N_{4} E_{q}}} \cdot\left[e^{-\tau E_{q}}+e^{-\left(N_{4}-\tau\right) E_{q}}\right]+\ldots .
\end{aligned}
$$

This expression is obviously different from that given in eq.(3.5). In particular, the first term in the r.h.s. in eq.(3.7) produces the 'shift upward' of the correlator with respect to the perturbative expression.

In our computations we monitored the total number of the Dirac plaquettes $N_{D P}^{(\mu \nu)}$ for every plane $(\mu \nu)$ and $N_{D P}=\max _{(\mu \nu)} N_{D P}^{(\mu \nu)}$. By a detailed investigation of the number and the location of Dirac plaquettes in the Coulomb phase, we observed pairs of Dirac sheets closed by periodic boundary conditions. In the extreme case of a minimal surface this is a pair of periodic Dirac sheets occupying parallel planes and having corresponding Dirac plaquettes with opposite signs. The appearance of periodically closed double Dirac sheet means that the number of the Dirac plaquettes in a given plane $(\mu \nu)$ has to be

$$
N_{D P}^{(\mu \nu)} \geq \frac{2 V_{4}}{N_{\mu} N_{\nu}} .
$$

For example, on a $12 \times 6^{3}$ lattice, the minimal number of Dirac plaquettes is $N_{D P}^{\min }=72$ if the Dirac sheets are timelike ('short' DDS), and $N_{D P}^{\min }=144$ if the Dirac sheets are spacelike ('long' DDS).

Figure 1 a shows a time history of the number of the Dirac plaquettes $N_{D P}$ on the $12 \times 6^{3}$ lattice at $\beta=1.1$. For every configuration the number $N_{D P}$ has been measured after the gauge fixing procedure. The number of configurations with Dirac sheets $\left(N_{D P} \geq 72\right)$ is $\sim 20 \%$. Long DDS $\left(N_{D P} \geq 144\right)$ are not as frequent as short DDS. However, as we shall see later the influence of the long DDS on the photon correlator is much stronger.

To convince ourselves that the appearance of the Dirac sheets is not an artifact of the small volume and/or small $\beta$-values we repeated these calculations on the lattices $12 \times 6^{3}$ and $24 \times 12^{3}$ at $\beta$-values between $\beta=1.1$ and $\beta=10$. We observed that the probability to find DDS shows rather weak dependence on the coupling. We observed also that the probability to find DDS increases slightly with increasing of the volume (e.g., at $\beta=2$ the number of configurations with DDS is $\sim 17 \%$ on the $12 \times 6^{3}$ lattice and $\sim 25 \%$ on the $24 \times 12^{3}$ lattice). In Figure [1 $1 \mathrm{~b}$ we show a time history of the number of the Dirac plaquettes $N_{D P}$ on the $24 \times 12^{3}$ lattice at $\beta=2$. This time history looks less 'noisy' as compared with that in Figure ta because there are less monopoles at $\beta=2$. 
In Figure 2a we show the transverse correlator $\Gamma(\tau ; \vec{p})$ with $\vec{p}=\left(0 ; \frac{2 \pi}{N_{2}} ; 0\right)$ at $\beta=1.1$ on the $12 \times 6^{3}$ lattice. Solid line corresponds to the lowest order perturbative expression given in eq.(3.5). Averaging over all configurations (filled circles) gives noticeable disagreement with the free photon case. On the other hand, excluding from the statistics configurations containing Dirac sheets gives an average correlator (opened circles) which fits nicely the zero-mass photon correlator. Squares represent a correlator obtained by averaging over configurations containing long Dirac sheets. The destructive influence of the Dirac sheets exhibits little changes with increasing $\beta$ and the lattice size. As an example we show in Figure 2 $\mathrm{b}$ the transverse correlator $\Gamma(\tau ; \vec{p})$ at $\beta=2$ on the $24 \times 12^{3}$ lattice.

The results presented in this section could be summarised by saying that the contribution of configurations with DDS 'spoils' the photon correlator and leads to a wrong dispersion relation inconsistent with the dispersion relation for the massless photon. Dirac sheets represent an example of lattice artifacts which can lead to a wrong interpretation of the results of numerical calculations.

\section{Zero-momentum correlator}

The (connected) zero-momentum correlator $\Gamma(\tau)$ is

$$
\Gamma_{\mu}(\tau)=\left\langle\mathcal{O}_{\mu}(\tau) \mathcal{O}_{\mu}(0)\right\rangle ; \quad \mathcal{O}_{\mu}(\tau)=\sum_{\vec{x}} \sin \theta_{x \mu}
$$

where $\tau$ is the separation in one of the four euclidian directions and $\vec{x}$ corresponds to the three complementary directions.

A usual way to define effective masses $m_{e f f}(\tau)$ is

$$
\frac{\cosh \left[m_{e f f}(\tau)\left(\tau+1-\frac{1}{2} N_{4}\right)\right]}{\cosh \left[m_{e f f}(\tau)\left(\tau-\frac{1}{2} N_{4}\right)\right]}=\frac{\Gamma_{\mu}(\tau+1)}{\Gamma_{\mu}(\tau)} .
$$

In the $S U(3)$ theory the large $-\tau$ behaviour of $m_{\text {eff }}(\tau)$ is supposed to describe the 'gluon mass' $m_{g}$ if $\tau$ is chosen along the 'temperature' direction $x_{4}$ and $\mu=1,2,3$ 6.

The definition of the effective mass in eq.(4.2) is such that for a correlator behaving like $\Gamma_{i}(\tau) \sim e^{-M \tau}+e^{-M\left(N_{4}-\tau\right)}$, one obtains $m_{e f f}=M$. However, on the periodic torus the gauge variant correlator $\Gamma_{i}(\tau)$ cannot be represented as a sum of exponents. Zero-momentum modes make their contribution to this correlator adding a nonzero positive constant as in eq.(4.7) [8]. As we shall see later this contribution cannot be discarded.

First, it is rather instructive to look at the distributions of the operators $\mathcal{O}^{(k)}(\tau)$ defined in eq.(4.1) and the correlations between different time-slices $\tau$, where the 
index $k$ stands for the $k^{t h}$ measurement. The width of the distribution of the zeromomentum operators is not small, and shows very weak dependence on the coupling constant in the interval between $\beta=1.1$ and $\beta=10.0$. As an illustration we show in Figure 3 a 'scatter-plot' of the operators $\mathcal{O}^{(k)}(\tau)$ with $\tau=1$ and $\tau=2$ at $\beta=2$ on a $24 \times 12^{3}$ lattice. For every measurement $k$ the operators $\mathcal{O}^{(k)}(\tau)$ can be represented as

$$
\mathcal{O}^{(k)}(\tau)=C^{(k)}+\delta \mathcal{O}^{(k)}(\tau)
$$

where $\delta \mathcal{O}^{(k)}(\tau)$ are small fluctuations about the constant contribution $C^{(k)}$. The width of the stripe in Figure 3 reflects the amplitude of these fluctuations.

It is worthwhile to note that correlations between operators $\mathcal{O}^{(k)}(\tau ; \vec{p})$ with nonzero momentum are very different from that for $\mathcal{O}^{(k)}(\tau)$, namely the scatter plot for $\mathcal{O}^{(k)}(\tau ; \vec{p})$ consists of a small 'blob' at the center of coordinates (is not shown).

It is not difficult to calculate the gauge variant correlator in the lowest approximation. The average of any functional $\Phi(\theta)$ is defined as

$$
\langle\Phi\rangle=\frac{1}{Z} \int\left[d \theta_{x \mu}\right] \Phi(\theta) \cdot e^{-S_{e f f}(\theta)}
$$

where $S_{e f f}=S+S_{g f}+S_{F P}, \quad S_{g f}=\frac{1}{\alpha g^{2}} \sum_{x}\left(\sum_{\mu} \bar{\partial}_{\mu} \sin \theta_{x \mu}\right)^{2}$, and $S_{F P}$ is the contribution from the corresponding Faddeev-Popov determinant. The choice $\alpha=$ 0 corresponds to the Lorentz gauge.

Lorentz gauge condition, does not exclude the appearance of the zero-momentum modes in the periodic volume. To calculate $\langle\Phi\rangle$ perturbatively one should keep the zero-momentum mode under control in the perturbation expansion. This can be achieved by repeating the Faddeev-Popov trick

$$
1=\left.J_{0} \int_{-\pi}^{\pi} \prod_{\mu=1}^{4} d \phi_{\mu} \exp \left\{-\frac{1}{\epsilon} \sum_{\mu}\left(\phi_{\mu}-\frac{1}{V_{4}} \sum_{x} \theta_{x \mu}\right)^{2}\right\}\right|_{\epsilon \rightarrow 0},
$$

and making the change of variables $\theta_{x \mu}=\phi_{\mu}+g A_{x \mu}$. The average functional $\langle\Phi\rangle$ can be now represented in the form

$$
\langle\Phi\rangle \sim \int_{-\pi}^{\pi}\left[d \phi_{\mu}\right] \int\left[d A_{x \mu}\right] \prod_{\mu=1}^{4} \delta\left(\sum_{x} A_{x \mu}\right) \Phi(\phi+g A) e^{-S_{\text {eff }}(\phi+g A)} .
$$

In eq. (4.6) one can safely expand in powers of $g^{2}$ and extend the limits of integration of $A_{x \mu}$ to $[-\infty ; \infty]$. The zero-momentum modes are not gaussian (the gauge action $S(\theta)$ does not depend on $\phi_{\mu}$ ), and the integration over $\phi_{\mu}$ in eq.(4.6) should stay compact.

Applying a perturbative expansion about the constant (zero-momentum) modes $\phi_{\mu}$ to calculate the zero-momentum correlators $\Gamma_{\mu}(\tau)$ one obtains 


$$
\Gamma_{\mu}(\tau) \sim\left\langle\sin ^{2} \phi_{\mu}\right\rangle \cdot\left(1-b_{\mu} g^{2}\right)+\frac{g^{2}}{2 V_{4}}\left\langle\cos ^{2} \phi_{\mu}\right\rangle \cdot \Gamma_{\mu}^{\prime}(\tau)
$$

where

$$
\begin{aligned}
\Gamma_{i}^{\prime}(\tau) & =\frac{g^{2} V_{3}^{2}}{2 V_{4}} \sum_{p_{4} \neq 0} \frac{e^{i \tau p_{4}}}{4 \sin ^{2} \frac{p_{4}}{2}} ; \quad i=1 ; 2 ; 3 ; \\
\Gamma_{4}^{\prime}(\tau) & =0 \quad \text { at } \quad \alpha=0
\end{aligned}
$$

and $V_{3}=N_{1} N_{2} N_{3}$. Coefficients $b_{\mu}$ represent tadpole contributions :

$$
b_{\mu}=\frac{1}{2 V_{4}} \sum_{p \neq 0} \frac{1}{\mathcal{K}^{2}}\left[1-\frac{\mathcal{K}_{\mu}^{2}}{\mathcal{K}^{2}}\right] ; \quad \mathcal{K}_{\mu}=2 \sin \frac{p_{\mu}}{2} .
$$

The coefficients $\left\langle\sin ^{2} \phi_{\mu}\right\rangle$ and $\left\langle\cos ^{2} \phi_{\mu}\right\rangle$ have been numerically calculated from distributions of the zero-momentum operators.

As an example we show in Figure 4 the correlator $\Gamma(\tau) \equiv \Gamma_{i}(\tau)$ at $\beta=2$ on a $12 \times 6^{3}$ lattice. Averaging over configurations without DDS (filled circles) gives a nice agreement with correlator defined in eq.(4.7) (solid line). The agreement becomes even better with increasing $\beta$. The increasing of the lattice size does change this conclusion. It is worthwhile to stress that this agreement is impossible without taking into account zero-momentum modes. Correlators defined on the configurations with DDS (diamonds and triangles) are very different from that defined on the configurations with DDS excluded.

\section{Conclusions}

We have studied the influence of Dirac sheets and zero-momentum modes on the gauge variant correlators $\Gamma(\tau ; \vec{p})$ with zero and nonzero momenta. All calculations have been performed on $12 \times 6^{3}$ and $24 \times 12^{3}$ lattices at different $\beta$ 's in the Coulomb phase.

We confirm our previous conclusion [4, f] about the role of the Dirac sheets : gauge copies which possess double Dirac sheets contribute to a wrong ('nonphysical') behaviour of the gauge variant photon propagators $\Gamma(\tau ; \vec{p})$ at $\vec{p}=0$ and $\vec{p} \neq 0$.

We have checked that with the increasing of the coupling from $\beta=1.1$ up to $\beta=10$ on a $12 \times 6^{3} \quad$ lattice and from $\beta=1.1$ up to $\beta=2$ on a $24 \times 12^{3}$ lattice the frequency of DDS does not change significantly. This frequency grows somewhat with the increasing of the volume. Because of configurations with DDS the numerically measured gauge variant correlators do not correspond to the free 
massless photons. Therefore, to avoid nonphysical conclusions one should exclude configurations with Dirac sheets out of consideration.

The contribution of the zero-momentum modes is of crucial importance for the interpretation of the zero-momentum correlators $\Gamma_{\mu}(\tau)$. These modes cannot be discarded at all $\beta$ 's and volumes we employed. A formal application of the definition of the effective mass $m_{\text {eff }}$ given in eq.(4.2) can produce a misleading interpretation.

\section{Acknowledgements}

One of us (VKM) gratefully acknowledge support obtained from the grant INTAS 96-0370 and the JINR Heisenberg-Landau grant. LDD is supported by PPARC under grants GR/L56329 and GR/L29927. Two of us (VKM and LDD) are pleased to thank the Physics Department of the University of Wales Swansea, where this work begun, for providing a stimulating working environment.

\section{References}

[1] K. Wilson, Phys. Rev. D10 (1974) 2445.

[2] V.N. Gribov, Nucl. Phys. B139 (1978) 1.

[3] A. Nakamura and M. Plewnia, Phys. Lett. 255B (1991) 274.

[4] V.G. Bornyakov, V.K. Mitrjushkin, M. Müller-Preussker and F. Pahl, Phys. Lett. 317B (1993) 596.

[5] V.K. Mitrjushkin, Phys. Lett. 389B (1996) 713.

[6] J.E. Mandula and M. Ogilvie, Phys. Lett. 185B (1987) 127.

[7] P. Goddington, A. Hey, J. Mandula and M. Ogilvie, Phys. Lett. 197B (1987) 191.

[8] V.K. Mitrjushkin, Phys. Lett. 390B (1997) 293.

[9] A. Cucchieri, Nucl. Phys. B508 (1997) 353.

[10] A. Cucchieri and T. Mendes, Nucl. Phys. (Proc. Suppl.) 63 (1998) 841.

[11] G. Damm, W. Kerler and V. K. Mitrjushkin, Phys. Lett. 433B (1998) 88. 
[12] A. M. Polyakov, Gauge fields and strings, Harwood Academic Publishers, (1987).

[13] T.A. DeGrand and D. Toussaint, Phys. Rev. D22 (1980) 2478.

[14] V. Grösch, K. Jansen, T. Jersák, C.B. Lang, T. Neuhaus and C. Rebbi, Phys. Lett. 162B (1985) 171.

\section{Figure captions}

Figure 1. Time history of $N_{D P}$ at $\beta=1.1$ on the $12 \times 6^{3}$ lattice (a) and at $\beta=2$ on the $24 \times 12^{3}$ lattice (b).

Figure 2. $\Gamma_{T}(\tau ; \vec{p})$ at $\beta=1.1$ on the $12 \times 6^{3}$ lattice (a) and at $\beta=2$ on the $24 \times 12^{3}$ lattice $(\mathbf{b})$.

Figure 3. Scatter-plot for operators $\mathcal{O}(\tau=1)$ and $\mathcal{O}(\tau=2)$ at $\beta=2$ on the $24 \times 12^{3}$ lattice.

Figure $1 . \quad \Gamma(\tau)$ at $\beta=2$ on the $12 \times 6^{3}$ 

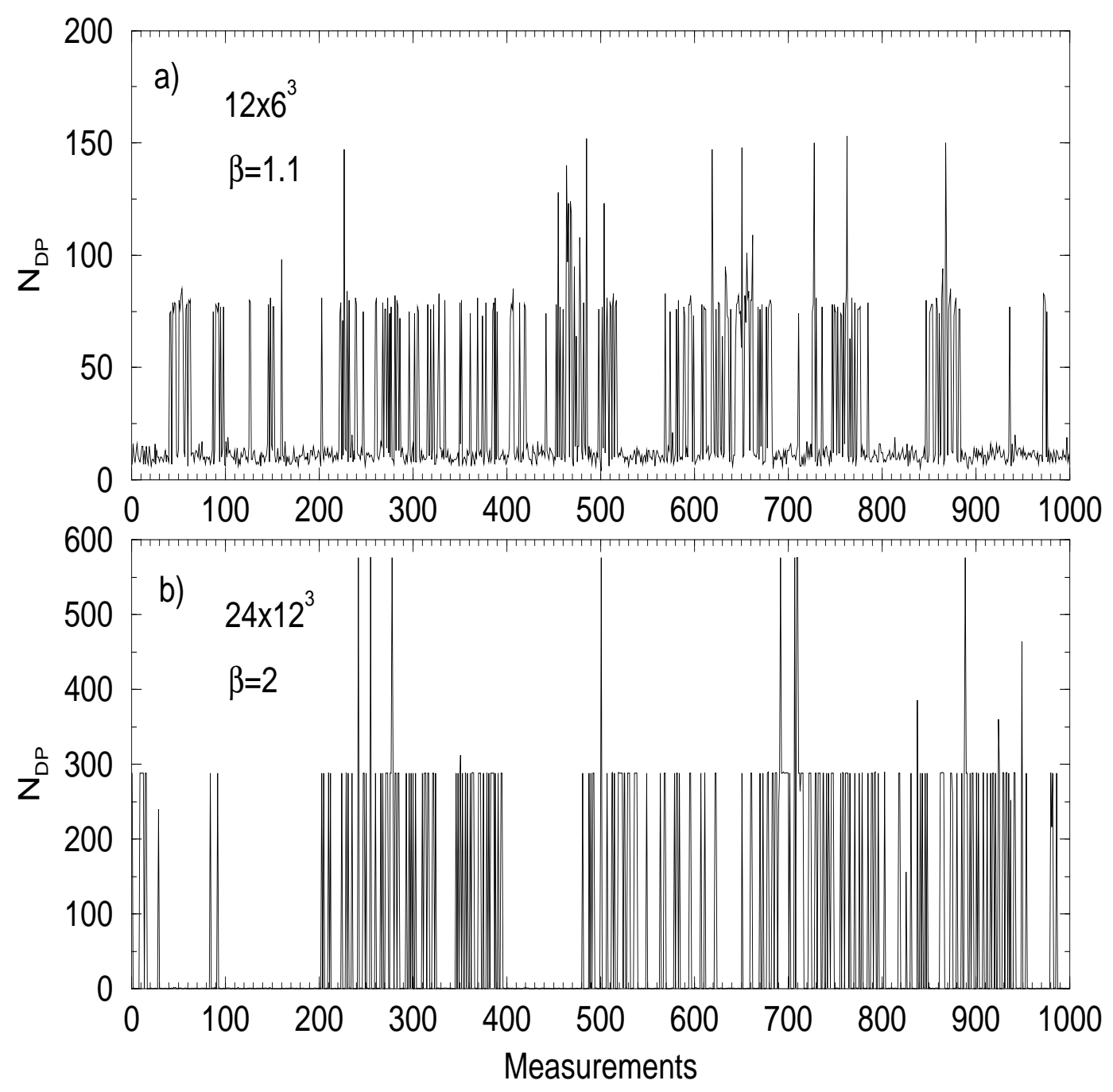

Figure 1: Time history of $N_{D P}$ at $\beta=1.1$ on the $12 \times 6^{3}$ lattice (a) and at $\beta=2$ on the $24 \times 12^{3}$ lattice (b). 

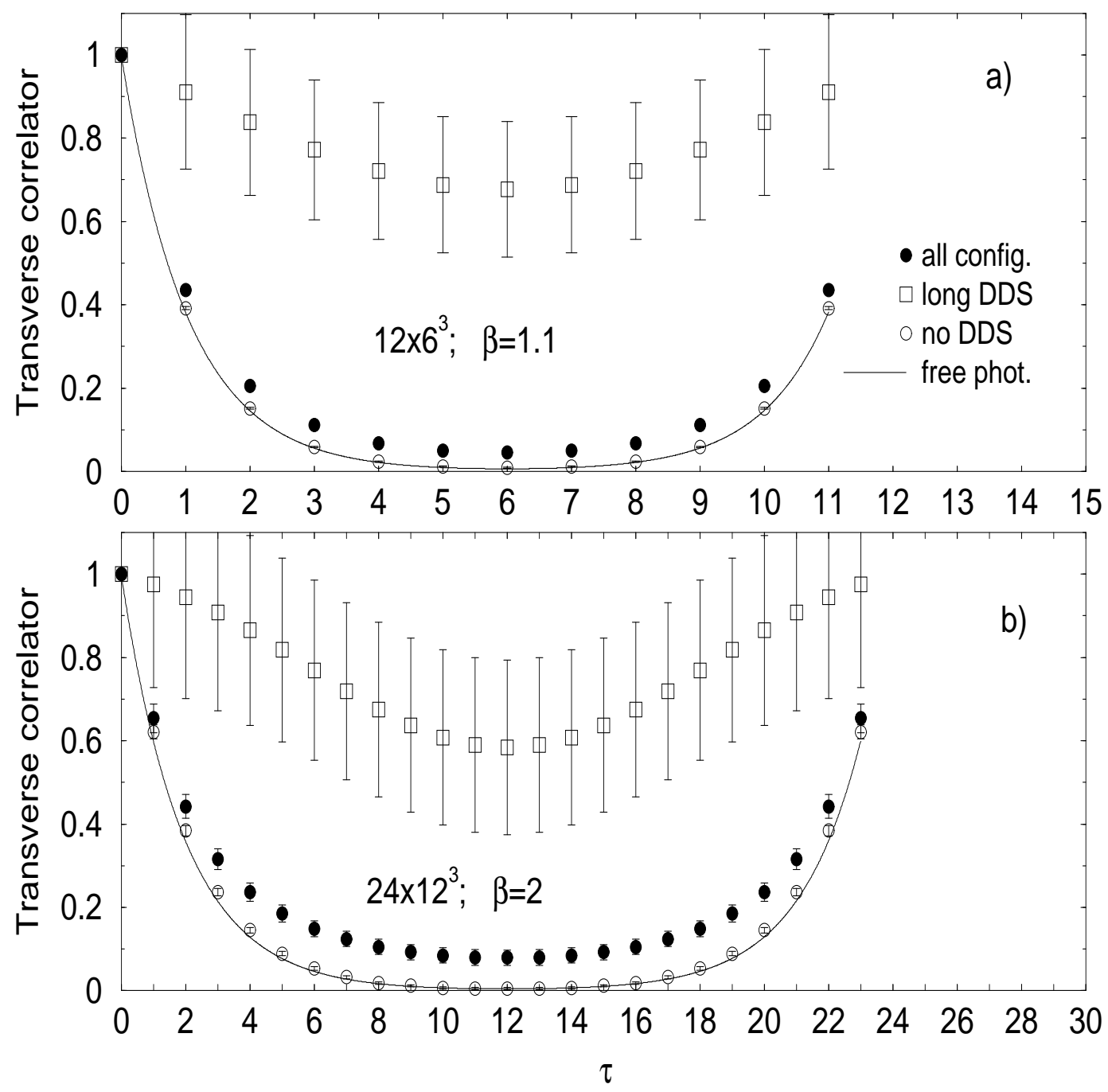

Figure $2: \Gamma_{T}(\tau ; \vec{p})$ at $\beta=1.1$ on the $12 \times 6^{3}$ lattice $(\mathbf{a})$ and at $\beta=2$ on the $24 \times 12^{3}$ lattice $(\mathbf{b})$. 


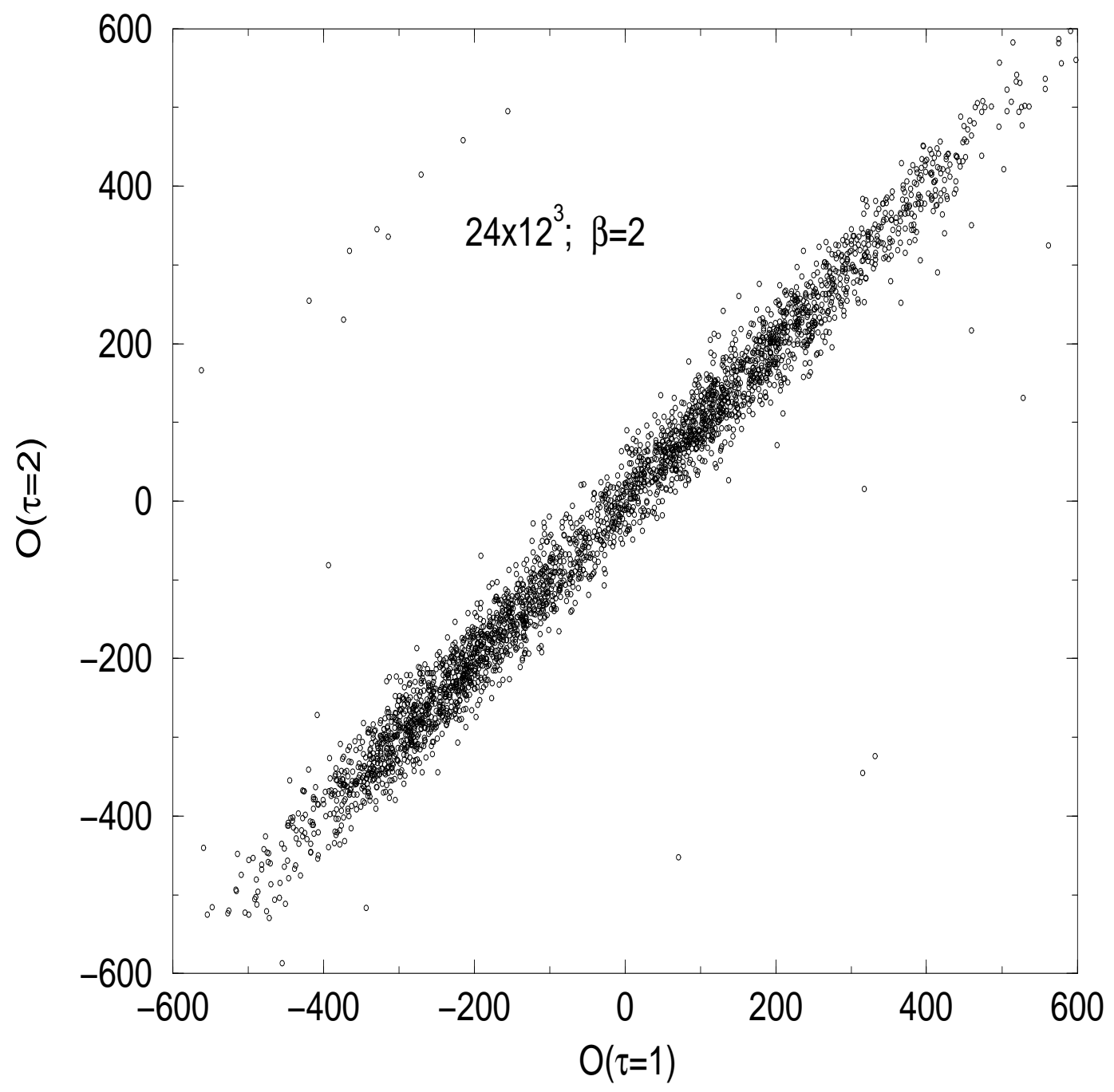

Figure 3: Scatter-plot for operators $\mathcal{O}(\tau=1)$ and $\mathcal{O}(\tau=2)$ at $\beta=2$ on the $24 \times 12^{3}$ lattice. 


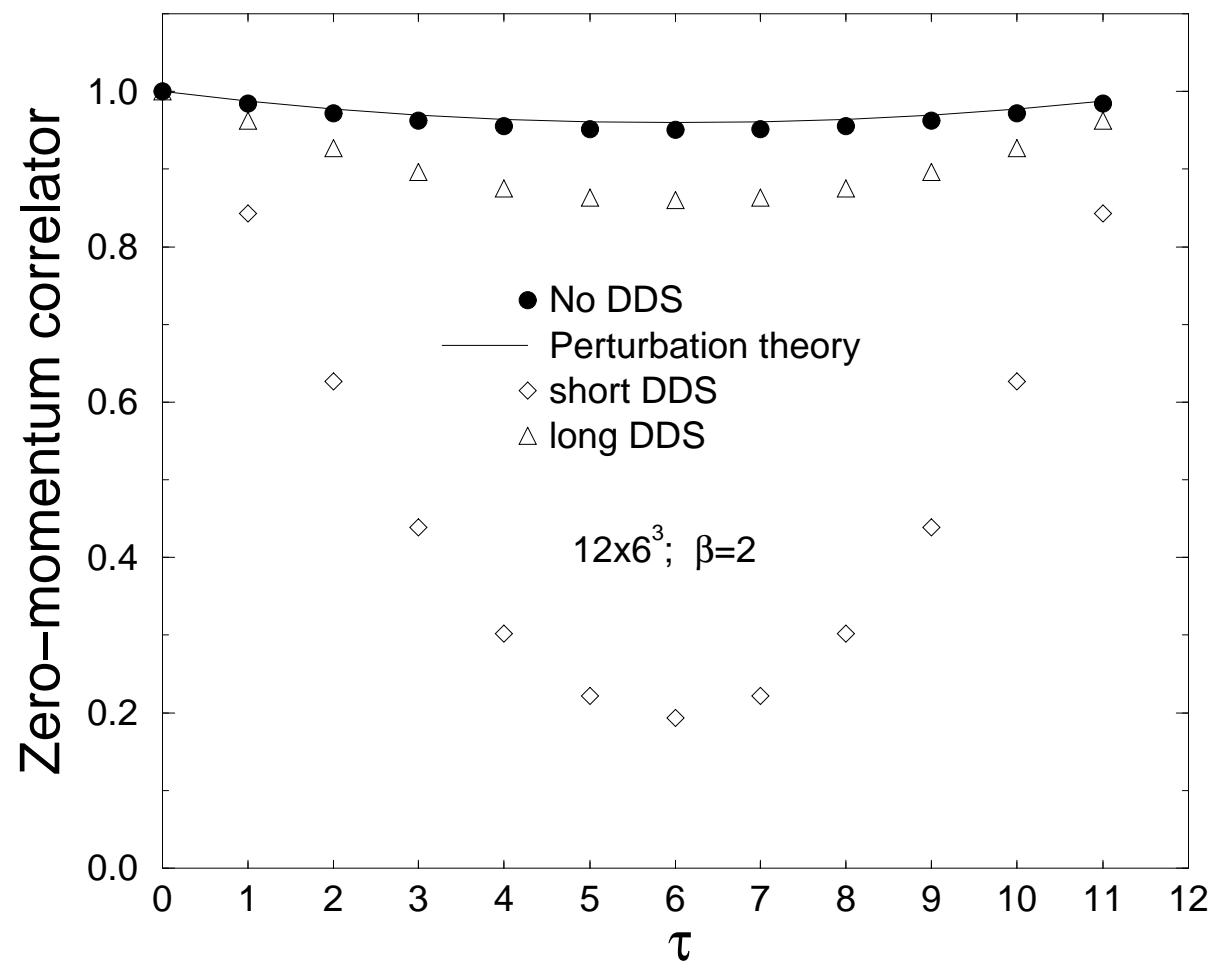

Figure 4: $\Gamma(\tau)$ at $\beta=2$ on the $12 \times 6^{3}$ lattice. 